\title{
Reactive Monolayers in Directed Additive Manufacturing - Area Selective Atomic Layer Deposition
}

\author{
Rudy J. Wojtecki ${ }^{*}$, Anuja DeSilva ${ }^{2}$, Noah Frederick Fine Nathel ${ }^{1}$, \\ Hosadurga Shobha ${ }^{2}$, Noel Arellano ${ }^{1}$, Alexander Friz ${ }^{1}$, and Greg Wallraff ${ }^{1}$ \\ ${ }^{1}$ IBM Almaden Research Center, 650 Harry Rd., San Jose, CA 95110, United States of America \\ ${ }^{2}$ IBM Semiconductor Technology Research, 257, Fuller Road, Albany, NY 12203, \\ United States of America \\ *rjwojtec@us.ibm.com
}

\begin{abstract}
Additive manufacturing generally describes a process of producing macro scale 3D-objects by the addition (or formation) of one patterned layer, of the overall object, onto a subsequent layer. In this fashion the target structure is produced in a layer-by-layer process. Selective area atomic layer deposition (SA-ALD) is, fundamentally, an additive manufacturing process where the layer-by-layer process occurs at the atomic level. A patterned surface is generated by exploiting disparate surface chemistries where a metal oxide is deposited in a targeted area. One method of enabling this process is through the use of organic monolayers that can form from a collection of well organized small molecules onto one surface and not another. These self-assembled monolayers (SAMs) can act as effective barriers to block the deposition of up to hundreds of thermal ALD cycles or can be used in combination with repair strategies to extend selective deposition capabilities. Recent efforts have focused on increasing the durability of these monolayer blocking layers. To this end we have designed SAM components that contain an aligning hydrogen bonding component that aid in directing the formation of well aligned monolayers as well as acting as a supramolecular cross-link. Furthermore, the components contain a photoactive diyne moiety that can be subsequently polymerized after irradiation to produce a robust ALD barrier. On structured surfaces this photoactive SAM achieved better deactivation properties than commercially available materials providing access to materials that produce a robust barrier in shorter formation times and withstand a great number of ALD cycles.

Keywords: Monolayers, Additive manufacturing, Photoactive monolayer, Self-assembled monolayer, Selective area atomic layer deposition, Atomic layer deposition
\end{abstract}

\section{Introduction}

Selective area atomic layer deposition (SA-ALD) is fundamentally an additive manufacturing process at the atomic scale. It is distinguished from the generally regarded additive manufacturing technologies by two key differences: (i) SA-ALD is a process that builds an object (usually films) one atomic layer at a time (ii) SA-ALD is a surface reaction that takes place from the gas phase. In combination with deactivation chemistry, on patterned surfaces, this layer-by-layer technique can be utilized to build a patterned films and can be extended to enable three-dimensional patterning schemes. SA-ALD offers the unique advantage of exploiting surface chemistry to deposit a material in a targeted area.

Monolayers, formed from a collection of well-organized small molecules, have been previously shown as effective barriers to block the deposition of hundreds of ALD cycles, or in combination with repair strategies to extend selective deposition capabilities directing this additive manufacturing process [1-3]. These techniques however, have only achieved with a 

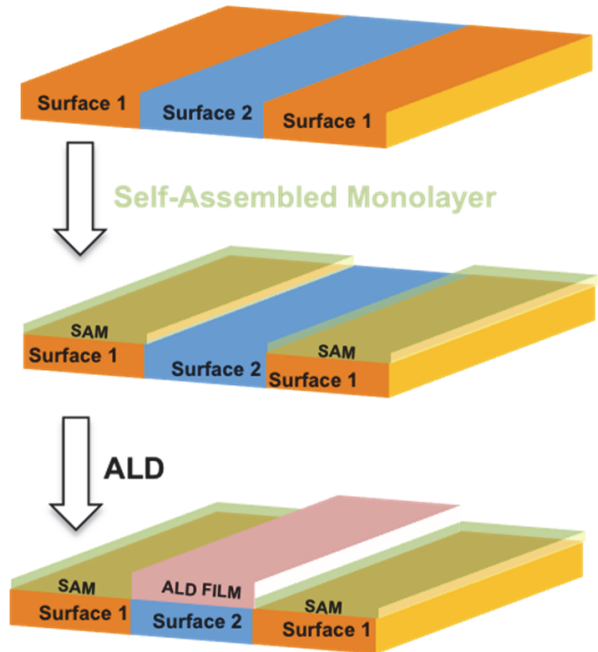

Fig. 1. Schematic illustration of the process flow for selective area atomic layer deposition. In the first step a monolayer component is introduced to a chemically dissimilar surface composed of two materials. The material adheres to one surface (SAM) deactivating it for a subsequent ALD step.

limited deposition of films and the use of a limited number of materials. For instance, most of the previous reports have detailed the use long aliphatic chains and varied the head group chemistry to selectively graft to different surfaces, $\mathrm{Si}-\mathrm{H}, \mathrm{SiO}_{2}$, metal oxides or bare metals [4] (Fig. $1)$.

This strategy, of introducing the smallest amount barrier composed of a single molecule layer of organic, is attractive as it offers the possibility of building structures through an additive manufacturing technique with high resolution. Achieving a selective deposition process is attracting particular interest from the semiconductor industry where it could eliminate a lithography step and therefore an alignment step which tend to introduce scaling errors (edge placement, overlay and critical dimension uniformity) that can all lead to device variation and ultimately impact performance. This would offer not only a significant cost savings but may also relax upstream design rules and enable access to non-traditional structures (e.g. 3D patterning).

The self-assembled monolayers (SAM) required deactivate an area from ALD deposition draw from well established head group chemistry enabling one to selectively deactivate a metal vs. a dielectric surface, or the opposite. Previous reports detailing SAALD processes have generally relied on long chain aliphatics that only have weak non-covalent Van der Waals interactions to direct the alignment of these components. As previously observed this may require extended periods of time to form a robust and pin-hole free monolayer film. However, there are methods of engineering components such that functional groups can be introduced to aid in alignment and ordering of the resulting monolayer surface [5].

Monolayer component (1) (Scheme 1) was designed to be a robust barrier for extended surface deactivation by incorporating supramolecular crosslinks and photoactive moieties within the backbone. Amide hydrogen bonding groups were introduced to aide in the alignment of and reduce monolayer surface formation time [6]. Furthermore, photoactive diacetylene functional groups were incorporated that are subsequently reacted to produce a robust polymeric barrier that deactivate surfaces for significantly more ALD cycles in comparison to unpolymerized monolayers [7]. These photopolymerizable monolayers were also evaluated as a blocking layer on planar structures with critical dimensions at and below $200 \mathrm{~nm}$ allowing us to quantify the limiting resolution of these monolayer masks.

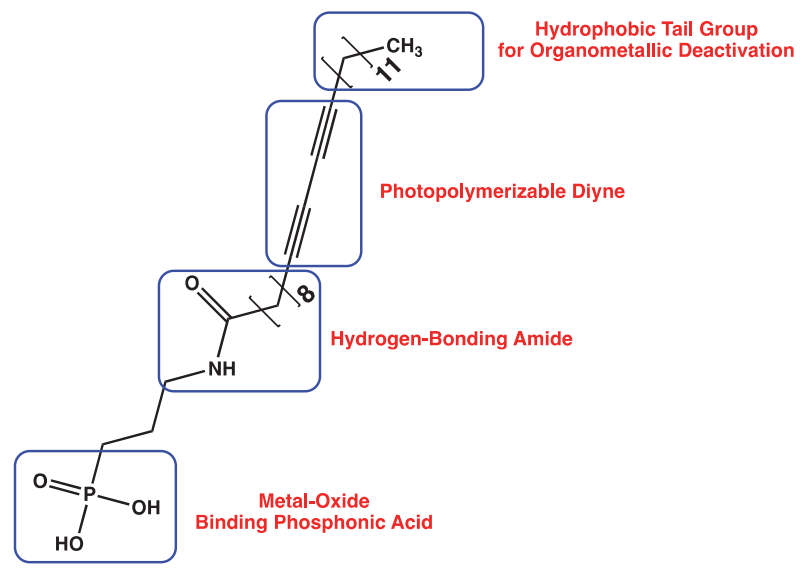

Scheme 1. Monolayer component (1) containing supramolecular aligning and photopolymerizable diyne moieties.

\section{Experimental}

Materials used in synthesis were purchased from Sigma-Aldrich. ${ }^{1} \mathrm{H}$ and ${ }^{13} \mathrm{C}$ NMR spectra were acquired in DMSO or $\mathrm{CDCl}_{3}$ using a Brüker $400-\mathrm{MHz}\left(100 \mathrm{MHz}{ }^{13} \mathrm{C}\right)$; chemical shifts $(\delta)$ are expressed in parts per million relative to TMS. XPS analysis performed on a Physical Electronics Quantum ESCA Microprobe, using a monochromated AlK $\alpha$ X-ray source at $1496.6 \mathrm{eV}$, 200 micron spot size throughout and charge 
neutralization. $1000 \mathrm{eV}$ survey spectra $(187 \mathrm{eV}$ pass energy, $0.8 \mathrm{eV} / \mathrm{step})$ and high resolution spectra $(58.7 \mathrm{eV}$ PE, $0.25 \mathrm{eV} / \mathrm{step})$ were acquired. High resolution spectra were referenced to $\mathrm{C}(1 \mathrm{~s})_{\max }=284.8 \mathrm{eV}$. Rutherford backscattering spectrometry using $\mathrm{MeV} \mathrm{He}+$ was used for measurment of film thickness and composition after atomic layer deposition. Static contact angle measurements were conducted using $50 \mu \mathrm{L}$ droplet volumes and a value generated through modeling software. A Cambridge NanoTech Savannah Atomic Layer Deposition System was used for all film deposition. Zinc oxide films were grown through a thermal process at $150{ }^{\circ} \mathrm{C}$ using diethyl zinc as the organometallic precursor and water as the oxidizer.

The patterned substrate was obtained from Albany Nanotech and is comprised of $\mathrm{Cu}$ line space patterns embedded in a dielectric layer prepared from octamethylcyclotetrasiloxane (OMCTS).

\subsection{Synthesis}

3-(Pentacosa-10,12-diynamido)propyl)phosphonic acid (DA, 1) was synthesized in three steps from commercially available diethyl (2-cyanoethyl)-phosphonate and 10,12-pentacosadiynoic acid (Scheme 2).

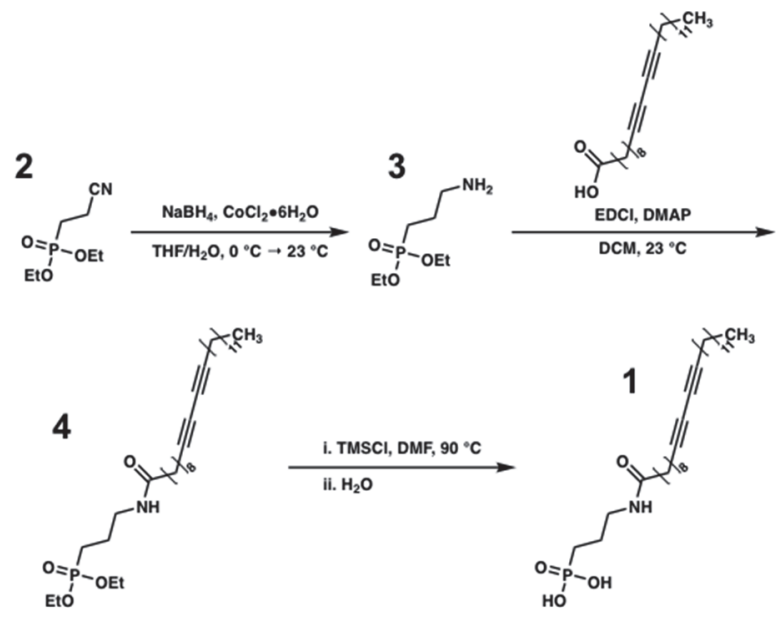

Scheme 2. Synthetic route to the preparation of $\mathbf{1}$.

\subsubsection{Diethyl (3-aminopropyl)phosphonate (2)}

Diethyl (2-cyanoethyl)phosphonate (3.24 g, $17.0 \mathrm{mmol}, 1.0$ equivalent) was reduced with sodium borohydride $(9.00 \mathrm{~g}, 237 \mathrm{mmol}, 14.0$ equivalents) and cobalt chloride hexahydrate $(0.81 \mathrm{~g}, 3.4 \mathrm{mmol}, 20 \mathrm{~mol} \%)$ in a $1: 1 \mathrm{THF} /$ water mixture $(65 \mathrm{~mL}, 0.1 \mathrm{M})$ at $0{ }^{\circ} \mathrm{C}$ and allowed to warm to $23{ }^{\circ} \mathrm{C}$ over 24 hours. $\mathrm{NH}_{4} \mathrm{OH}(1 \mathrm{~L}$, $28 \%$ in $\mathrm{H}_{2} \mathrm{O}$ ) was added and stirred for $1 \mathrm{~h}$. The aqueous phase was extracted with DCM $(4 \times 200$ $\mathrm{mL}$ ), which was dried over $\mathrm{MgSO}_{4}$ and concentrated in vacuo to give diethyl (3-aminopropyl)phosphonate (1.51 g, $7.74 \mathrm{mmol}$, $46 \%$ crude yield). This was carried forward without further purification. ${ }^{1} \mathrm{HNMR}(400 \mathrm{MHz}$, $\left.\mathrm{CDCl}_{3}\right): \delta 4.10(\mathrm{q}, J=6.9,4 \mathrm{H}), 2.77(\mathrm{t}, J=6.6,2$ H), $1.84-1.66(\mathrm{~m}, 4 \mathrm{H}), 1.32(\mathrm{t}, J=7.1,6 \mathrm{H})$.

2.1.2. Diethyl (3-(pentacosa-10,12-diynamido)propyl)phosphonate (3)

1.0 Equivalent of $2(0.85 \mathrm{~g}, 4.32 \mathrm{mmol})$ was coupled with 10,12-pentacosadiynoic acid $(1.62 \mathrm{~g}$, $4.32 \mathrm{mmol}, 1.0$ equivalent $)$ using EDCI $(0.75 \mathrm{~g}$, $4.8 \mathrm{mmol}, 1.1$ equivalents) in the presence of DMAP (0.03 g, $0.22 \mathrm{mmol}, 5 \mathrm{~mol} \%)$ in dichloromethane $(12 \mathrm{~mL}, 0.4 \mathrm{M})$ over $24 \mathrm{~h}$. The reaction was washed with $\mathrm{H}_{2} \mathrm{O}(2 \times 200 \mathrm{~mL})$. The organic phase was dried over $\mathrm{MgSO}_{4}$ and then concentrated in vacuo. The crude product was purified by column chromatography $(50 \%$ EtOAc/Hexanes $\rightarrow 5 \% \mathrm{MeOH} / \mathrm{DCM})$ to generate diethyl (3-(pentacosa-10,12-diynamido)propyl)phosphonate (C-2, $0.63 \mathrm{~g}, 1.17 \mathrm{~mol}, 27 \%$ yield). ${ }^{1} \mathrm{HNMR}\left(400 \mathrm{MHz}, \mathrm{CDCl}_{3}\right): \delta 6.05(\mathrm{~s}, 1 \mathrm{H}), 4.10$ (q, $J=7.5,4 \mathrm{H}), 3.35$ (q, $J=6.3,2 \mathrm{H}), 2.24(\mathrm{t}, J$ $=6.9,4 \mathrm{H}), 2.16(\mathrm{t}, J=7.4,2 \mathrm{H}), 1.88-1.71(\mathrm{~m}, 4$ $\mathrm{H}), 1.56(\mathrm{~m}, 37 \mathrm{H}), 0.88(\mathrm{t}, J=6.9,3 \mathrm{H})$.

\subsection{3. (3-(Pentacosa-10,12-diynamido)propyl) phosphonic acid (4)}

1.0 Equivalent of $3(0.32 \mathrm{~g}, 0.60 \mathrm{mmol})$ was deprotected by forming the trimethylsilyl phosphonate in-situ with chlorotrimethylsilane (3.89 g, $35.8 \mathrm{mmol}, 60.0$ equivalents) in DMF (5 $\mathrm{ml}, 0.12 \mathrm{M}$ ) at $90{ }^{\circ} \mathrm{C}$ over $24 \mathrm{~h}$. Hydrolysis of the trimethylsilyl ester with water produced the desired product DA ( $0.25 \mathrm{~g}, 0.50 \mathrm{~mol}, 84 \%$ yield). The crude DA was purified by filtering the crude solid, rinsing the filtered solid with $\mathrm{H}_{2} \mathrm{O}(5 \mathrm{~mL})$, and trituration with DCM $(3 \times 5 \mathrm{~mL})$. ${ }^{1} \mathrm{HNMR}$ (400 MHz, DMSO- $\left.d_{6}\right): \delta 3.04$ (q, $J=5.8,4 \mathrm{H}$ ), $2.27(\mathrm{t}, J=6.8,4 \mathrm{H}), 2.02(\mathrm{t}, J=7.7,2 \mathrm{H}), 1.63$ $1.07(\mathrm{~m}, 36 \mathrm{H}), 0.85(\mathrm{t}, J=6.6,3 \mathrm{H})$.

\subsection{Monolayer film preparation}

A $500 \mathrm{~nm} \mathrm{Cu}$ film was thermally evaporated onto a four inch reclaimed silicon wafer using a circular shadow mask that protected a portion of the native $\mathrm{SiO}_{2}$ surface from $\mathrm{Cu}$ deposition. Immediately prior to monolayer solution 
exposure the $\mathrm{Cu}$ surface was cleaned with a brief oxygen plasma (30 s) to remove any organic contaminants from the surface. The wafer was soaked in a $0.5 \mathrm{mg} / \mathrm{mL}$ filtered solution of 1 in dimethyl sulfoxide at $65^{\circ} \mathrm{C}$ for $24 \mathrm{~h}$, then rinsed with isopropanol and dried. The contact angle on the $\mathrm{Cu}$ surface was measured to be $104^{\circ}$.

\section{Results and discussion}

3.1. Design \& synthesis of monolayer components

Monolayers composed of long aliphatic chains such as octadecyl phosphonic acid have been reported to deactivate the surface of metal oxides from the ALD deposition of zinc oxide using a thermal process where the cycle process first introduces dimethyl zinc followed by water for the other half cycle. With this monolayer surface inhibition the surface was deactivated for up to 600 cycles of ALD growth. Motivated to explore the realm of materials that could extend this period of deactivation we designed a component containing a supramolecular hydrogen bonding group to aid in the alignment and formation of the monolayer and a diyne component where after the monolayer formation it could be subsequently be reacted to produce a polymeric species at the surface. We designed this molecule $\mathbf{1}$ and synthesized it according to Scheme 2. Briefly, 1 was synthesized in three steps from commercially available diethyl (2-cyanoethyl)phosphonate and 10,12-pentacosadiynoic acid. Where (2-cyanoethyl)phosphonate was first reduced to diethyl (3-aminopropyl)phosphonate with sodium borohydride and cobalt chloride hexahydrate. The resulting amine was carried forward without further purification and coupled with 10,12-pentacosadiynoic acid using 1-ethyl-3-(3-dimethylaminopropyl)carbodiimide $(\mathrm{EDCl})$ in the presence of 4-dimethylaminopyridine (DMAP) in dichloromethane generating diethyl(3-(pentacosa-10,12-diynamido)propyl)phosphonate that was subsequently deprotected by forming the trimethyl silyl phosphonates in situ with chlorotrimethylsilane (60 equiv.) in DMF at $90{ }^{\circ} \mathrm{C}$ and hydrolysis with water to yield $\mathbf{1}$.

Using this material monolayers were produced on copper surfaces by first dissolving this material in dimethylsulfoxide (DMSO) and immersing a freshly cleaned copper film (using a brief oxygen plasma) in the solution for a period of $24 \mathrm{~h}$. This extended immersion time may not be completely necessary as it was observed as soon as a droplet of DMSO containing 1 was added to the copper surface the droplet would immediately bead up and dewet from the $\mathrm{Cu}$ surface. After monolayer formation the contact angle on the $\mathrm{Cu}$ surface was measured to be $104^{\circ}$, indicating formation of a well-assembled SAM. After these monolayer films were prepared they were first baked under $\mathrm{N}_{2}$ at $180{ }^{\circ} \mathrm{C}$ for $5 \mathrm{~min}$. This baking procedure dehydrates the surface and drives the formation of a more stable phosphonate ester. The wafer was split into two halves. One half was irradiated with $254 \mathrm{~nm}$ light for $5 \mathrm{~min}$. The contact angle of the monolayer post-irradiation was $101^{\circ}$, indicating negligible desorption of the SAM. The carboxylic acid functionality of the starting material containing the diyne was previously reported to undergo a removal of the monolayer generating a hydrophilic surface after a long enough exposure to UV highlighting the structural requirement to have a different head group chemistry [8]. This behavior was not observed when photopolymerizing a similar diyne surface [7].

The curing process, which generates this exceptional barrier to ALD is a topochemical polymerization, in which molecules react in the solid state via initiation of an active site and auto-

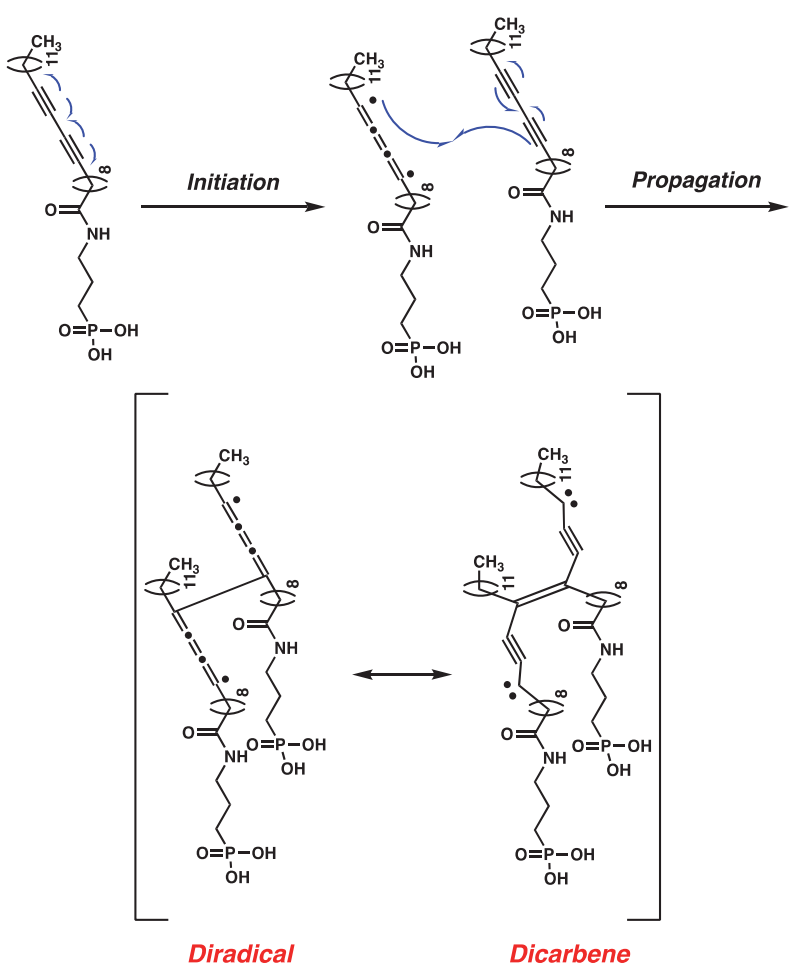

Scheme 3. Photopolymerization mechanism of $\mathbf{1}$. 
propagation through a preordered lattice. In this case, the diacetylene units are the active sites, which are ordered by the chemical interaction of the phosphonic acid head groups with the copper substrate and the hydrogen bonding of the adjacent amides. Initiation occurs when a diacetylene unit is exposed to ultraviolet light, exciting electrons into a higher energy state and generating a reactive diradical species. This radical species reacts with surrounding diacetylene molecules to propagate the polymerization. During this phase, the oligomers formed are butatrienes. It has been reported that when the degree of polymerization exceeds 6 , the reactions mechanism predominantly switches and the active species becomes a triplet carbene. Further polymerization therefore generates an ene-yne backbone as opposed to a butatriene (Scheme 3).

\subsection{Blanket ALD studies}

The two samples baked and photoirradiated were then submitted thermal ALD of $\mathrm{ZnO}$ at $150{ }^{\circ} \mathrm{C}$ using diethyl zinc and water for each half cycle. On surfaces not containing deactivating chemistry film thickness after each cycle was measured as ca. $0.12 \mathrm{~nm}$. Coupons of each half were removed from the ALD chamber after every 100 cycles for a total of 10 coupons or 1000 ALD cycles. These coupons were then characterized by Rutherford backscattering spectrometry (RBS) to measure surface composition. The $\mathrm{ZnO}$ composition increased immediately on the $\mathrm{SiO}_{2}$ surface, consistent with the growth of $\mathrm{ZnO}$ film (Table 1).

Table 1. $\mathrm{ZnO}$ film growth composition and thickness on $\mathrm{Si}$ surface. Uncertainties in atomic $\%$ are $+/-3 \%$.

\begin{tabular}{crrc}
\hline $\begin{array}{c}\text { ALD } \\
\text { Cycles }\end{array}$ & $\begin{array}{r}\text { Zn } \\
\text { at. } \%\end{array}$ & $\begin{array}{c}\text { O } \\
\text { at. } \%\end{array}$ & Thickness $(\AA)$ \\
\hline 200 & 46 & 54 & $195 \pm 10$ \\
400 & 50 & 50 & $449 \pm 20$ \\
600 & 50 & 50 & $749 \pm 50$ \\
800 & 49 & 51 & $1100 \pm 100$ \\
1000 & 49 & 51 & $1385 \pm 100$ \\
\hline
\end{tabular}

On copper with the non-photoirradiated SAM, the concentration of $\mathrm{ZnO}$ was undetectable up to 800 cycles, where at 1000 cycles, $47 \AA$ of $\mathrm{ZnO}$ was detected. On copper with the photoirradiated

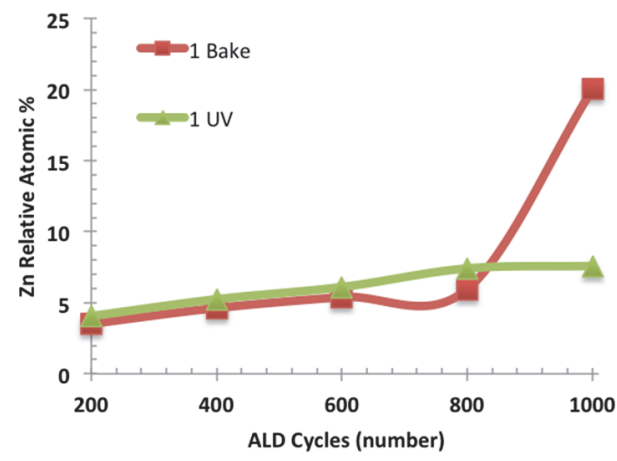

Fig. 2. XPS characterization of monolayer films composed of (1) on $\mathrm{Cu}$ UV cured vs. baked.

SAM, no $\mathrm{ZnO}$ was detected up to 1000 cycles, particle induced X-ray emission (PIXE) was also used to confirm these results in the RBS where $\mathrm{Zn}$ was only detected on the higher number of cycles on the deactivated $\mathrm{Cu}$ surfaces. In addition we used X-ray photoelectron spectroscopy to characterize these coupons which showed a consistent result (Fig. 2). It can be concluded that, while the photoirradiated SAM is a strong barrier to ALD, the barrier formed upon photoirradiation improves ALD deactivation.

\subsection{Patterned ALD studies}

We then turned our attention to evaluate the performance of this material in comparison to a commercially available octadecylphosphonic acid (ODPA) on a patterned surface. Coplanar surfaces of $\mathrm{Cu}$ embedded in an OMCTS dielectric
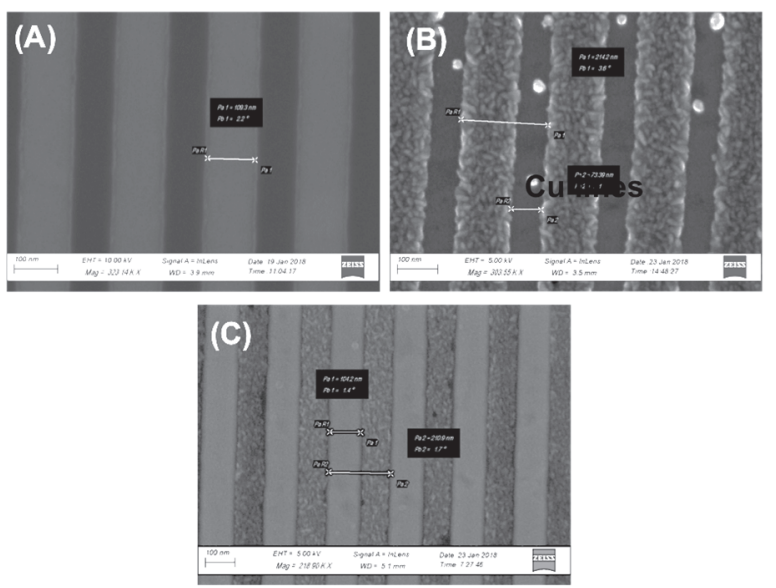

Fig. 3. SEM Micrographs of patterned $\mathrm{Cu}$ surfaces with dielectric interspaces. (A) Surfaces before monolayer protection and ALD. Darker colored lines $\mathrm{Cu}$, OMCTs dielectric lighter color (B) $\mathrm{ZnO}$ growth observed on the dielectric surface after 200 ALD cycles using ODPA as a deactivating surface (C) using (1) after photocuring showing a significant difference in overgrowth. 
that behaved as similar to a native $\mathrm{SiO}_{2}$ layer in our coupon deactivation studies. Using the same ALD recipe and curing conditions for 1 we compared the performance of a ODPA deactivated surface. It is important to note that during this experiment we observed the formation of $\mathrm{Cu}$ dendrites that should not be confused with the nucleation of $\mathrm{ZnO}$ particles on the $\mathrm{Cu}$ surfaces. These are readily apparent in the SEM because of their brighter color consistent with an increase in the secondary electron yield of these structures. After 200 cycles it was readily apparent that on $\mathrm{Cu}$ lines measuring ca. $110 \mathrm{~nm}$ across before 200 cycles of thermal ZnO ALD. After 200 cycles the commercially available ODPA resulted in a selective deposition but 31 $\mathrm{nm}$ of $\mathrm{ZnO}$ overgrowth onto the $\mathrm{Cu}$ surfaces was observed (Fig. 3B). However, when $\mathbf{1}$ is used in the same conditions significantly less overgrowth was observed (ca. $4 \mathrm{~nm}$ ). In addition, we observed when ODPA was used the $\mathrm{ZnO}$ deposited produced continuous films. 1 was observed to also inhibit some portion of the OMCTS surfaces as well and $\mathrm{ZnO}$ was not observed to produce a continuous film.

\section{Conclusion}

Hydrophobic surfaces composed of long chain aliphatic chains are effective at deactivating thermal $\mathrm{ZnO}$ ALD film recipes. The introduction of supramolecular interactions may aid in decreasing the formation time and alignment of the components. Photoactive diyne moieties enable access to surfaces that can be subsequently photopolymerized to yield a more robust barrier than a typical commercially available material without these features. On structured surfaces the these materials extended and enhanced the deactivation process though this may have also lead to some deactivation of the intended deposition site (OMCTS surface). Future work is aimed at extending the versatility of this material and its selectivity in other thermal ALD film compositions.

\section{Acknowledgement}

We would like to thank Andrew Kellock and Andy Tek for the helpful discussions and insights in the RBS and XPS characterization.

\section{References}

1. C. Prasittichai, K. L. Pickrahn, F. S. M. Hashemi, D. S. Bergsman, and S. F. Bent, $A C S$ Appl. Mater. Interfaces, 6 (2014) 17831.

2. X. Jiang and S. F. Bent, J. Phys. Chem. C, 113 (2009) 17613.

3. R. C. Kim, P. C. McIntyre and S. F. Bent, App. Phys. Lett., 84 (2004) 4017.

4. R. Chen and S. F. Bent, Chem. Mater., 18 (2006) 3733.

5. R. Valiokas, M. Östblom, S. Svedhem, S.C.T. Svensson, and B. Liedberg, J. Phys. Chem. B, 106 (2002) 10401.

6. M. A. Ramin, G. L. Bourdon, K. Heuze, M. Degueil, T. Buffeteau, B. Bennetau, and Luc Velltini, Langmuir, 31 (2015) 2783.

7. F. Li, E. Shishkin, M. A. Mastro, J. K. Hite, C. R. Eddy, J. H. Edgar, and T. Ito, Langmuir, 26 (2010) 10725.

8. G. Kwak, M. Seol, Y. Tak, and K. Yong, J. Phys. Chem. C, 113 (2009) 12085. 